\title{
PROGRAMA DE SOCIALIZACIÓN ECONÓMICA EN GESTIÓN NEGOCIOS Y RELACIONES DE CONFIANZA EN POBLADORES DE ZONA URBANA Y RURAL EN CARAZ, HUAYLAS, ANCASH
}

\author{
ECONOMIC SOCIALIZATION PROGRAM IN BUSINESS MANAGEMENT AND \\ TRUST RELATIONSHIPS IN PEOPLE FROM URBAN AND RURAL AREAS IN \\ CARAZ, HUAYLAS, ANCASH
}

Mildred Paredes T.*, Héctor Hernández V., Renato Santivañez O., Miguel iturrizaga A., Luis Vicuña P., Ronald

Castillo B., César Zamalloa S.

Universidad Nacional Mayor de San Marcos, Lima, Perú

(RECIBIDO 06-08-2013, ACEPTADO 20-10-2013)

\begin{abstract}
RESUMEN
Investigación de tipo experimental, diseño cuasiexperimental, con el objetivo de observar el efecto del Programa de socialización económica sobre la gestión de unidades productivas y relaciones de confianza en negocios en un grupo de 24 colaboradores mayores de 18 años voluntarios de zona urbana y rural de Caraz. Se diagnosticó y capacitó sobre los principios de la administración: Dirección, Coordinación, Organización, Planificación y Comunicación. Se hizo medición antes y después en la Disposición hacia las Relaciones de Confianza en los Negocios con una Escala tipo Likert de cuatro factores: a) Competencia b) Preocupación por el otro, c) Equidad, y d) Visión de sí mismo. La confiabilidad del instrumento es de 0,74 y la validez se estableció por el método de contenido. Se encontró $47 \%$ en conocimientos de gestión de negocios, destaca el principio de Organización y de Dirección, en cambio con valores bajos en las dimensiones de Evaluación, Estrategias y Elaboración de objetivos. Se encuentra una tendencia alta en la disposición a confiar y tener confianza en un socio, confianza que mejora solo por el factor Competencia y disminuye por el factor Equidad por acción del Programa de Socialización Económica para las relaciones de confianza en pobladores de zona urbana y rural asociados en Caraz, Huaylas, Ancash.
\end{abstract}

Palabras clave: relaciones de confianza, socialización económica, preocupación por el otro, gestión de negocios, competencia

\begin{abstract}
Experimental research, quasi-experimental design that aims to observe the effect of economic socialization program on the management of production units and business relationships of trust in a group of 24 volunteers over 18 years old from urban and rural area of Caraz. Was diagnosed and trained on the principles of administration: Management, Coordination, Organization, Planning, and Communication. Measurement was made before and after the disposition towards trust relationships in the business, with a Likert scale of four factors:
\end{abstract}

* Docente Principal, Facultad de Psicología UNMSM. E-mail: mildredparedestarazona@gmail.com 
PRogRAmA de SOCIALIZACIÓN ECONÓMICA EN GESTIÓN NEGOCIOS Y RELACIONES DE CONFIANZA...

a) Competition b) Concern for the other, c) Equity, d) View of himself. The reliability of the instrument was 0.74 and validity established by the method of content. We found $47 \%$ in business management skills, emphasizes the principle of Organization and Management, in contrast with low values in the dimensions of Evaluation, Development Strategies and Objectives.

It is a high tendency in the willingness to trust and have confidence in a partner, who only enhances by Competition Factor and decreases by a factor of Equity because of Economic Socialization Program for relations of trust in residents of urban and rural partners Caraz, Huaylas, Ancash.

Keywords: trust relationships, economic socialization, concern for others, business management, competition

\section{INTRODUCCIÓN}

Las relaciones de confianza para la gestión de negocios es una dimensión psicológica que transversalmente interviene proporcionando vínculos positivos en las interacciones personales y económicas, y por consiguiente, en las relaciones institucionales u organizacionales. Estas son adquiridas en la socialización económica que todo individuo aprende en sus interacciones sociales, las que se encuentran delimitadas por la cultura mediante normas, pautas y formas de comportamiento de sus transacciones económicas.

Es de importancia tomar las referencias de Bowlby (1999), quien señala que las relaciones de confianza se instituyen en la edad de lactancia en la aproximación y alejamiento durante la interacción madre-hijo, con la presencia afectiva y de autoridad del padre. Relación identificada como estado psicológico que se va construyendo al igual que el Estilo de Vida según Adler (Paredes, 2010). Con respeto a los aspectos éticos y morales como producto del desarrollo psicológico, Kohlberg describe la Teoría del Razonamiento moral en tres niveles en 1968; siendo en el Nivel III en el que se logra los principios morales autónomos como la Moralidad completamente internalizada, en donde se reconocen los conflictos entre estándares morales y se hacen juicios propios con base a criterios como lo correcto, la igualdad y la justicia, los que combinan con la confianza. Este nivel se alcanza al menos a los 13 años, más comúnmente en la adultez joven (Papalia, Sterns, Feldman y Camp, 2009).

En el ámbito organizacional y empresarial, Guillen (2006) puntualiza que la "virtud de la confianza en los demás" se manifiesta en el comportamiento de mayor o menor seguridad que tiene la persona al tomar una decisión respecto a la cooperación de los involucrados en el logro del objetivo que se busca. Así, "quien confía en los demás, se fía de los implicados, quienes harán lo que les corresponda en ese proceso de decisión", situación correspondiente a una conducta de participación.

Paredes, Hernández, Héctor, Vicuña y González (2012) concluyen que las actitudes hacia la Interdependencia empresarial para el emprendimiento en pobladores de la ciudad de Caraz son positivas aun cuando "no afecta el Programa de socialización económica en interdependencias empresariales e institucionales para orientar el 
emprendimiento del turismo rural-vivencial en Caraz, Ancash", en el que atribuyeron que sus actividades en el sector turismo no les reedita utilidad.

Paredes, Hernández, Vicuña, Merino y cols. (2010) identifican en una muestra de pobladores rurales del Caserío de Queral, Caraz, que sus actitudes hacia el empoderamiento son de tendencia positiva y que El Programa de Alfabetización Económica afecta las Conductas de Emprendimiento: Manejo de recursos económicos y humanos, Disposición a la innovación, Aprovechamiento de la oportunidad, Toma de decisiones en condiciones de incertidumbre, Administración del caos. Asimismo, difieren las actitudes hacia el empoderamiento según el género a favor de los varones, la condición de función o rol, y la edad en mayores.

En el ámbito de los negocios se hace necesario la cooperación y la competitividad. No se concibe la competitividad en los negocios sin una estrategia que considere al otro, ya sea en una posición de competidor, cliente, proveedor o persona que pueda asumir el rol de socio con el propósito de emprender o posesionar el negocio. Es así, por ejemplo, que en la política actual se vislumbra el programa del gobierno peruano: "Un pueblo, un producto", coordinado por el Mincetur y auspiciado por el BID y el gobierno de Japón; sin embargo, esta se dificultaría si no se consolidan las iniciativas de los pequeños empresarios de los distritos tanto de zonas rurales como urbanas. Es a partir de esto que el conocimiento de las variables psicológicas y psicosociales, que sustentan el comportamiento, permite retroalimentar a la población para la toma de sus propias decisiones a fin de encaminar su crecimiento económico en base a sus propias competencias y productos mediante su emprendimiento y posicionamiento.

Hay evidencia que la percepción hacia valores como lo que es correcto, la igualdad o la justicia centran la relación de confianza, pilar en la formación y fortalecimiento de todo negocio; sin embargo, a pesar que los colaboradores poseen actitudes hacia el emprendimiento tendientes a ser positivas, hay renuencia a formar alianzas económicas u optar como estrategia una gestión que evidencie beneficio sostenible, de manera específica a establecer un negocio con un socio o partner.

Luego, es así que el rechazo a constituir estas relaciones se expresan como "miedo a que les roben", mientan y que, consiguientemente, se apropien de sus ideas para que luego se separen, copien su manera de trabajar y, sobre todo, que se queden con su "plata", verbalizan "mejor solo que mal acompañado". Es así que, en el contexto de esta realidad y de las intervenciones que desde la Psicología se realizan para modificar aspectos de interés en un determinado grupo, se plantea la siguiente pregunta de investigación:

¿Cuál es el efecto del Programa de Socialización económica en gestión de negocios en las relaciones de confianza en pobladores de zona urbana y rural asociados en Caraz, Huaylas, Ancash?

El diseño de la investigación es cuasiexperimental empleándose, como parte del Programa de Socialización económica en gestión y las relaciones de confianza, talleres de capacitación en procesos administrativos instrumentado por casos y 
dinámicas de grupo para que cognitivamente los participantes puedan observar un modelo de un negocio o proyecto de negocios productivo de un bien o servicio en torno a un producto propio del lugar.

Como variable independiente (VI) es la intervención, mediante el Programa de Capacitación con talleres que permite una intervención vivencial en módulos guiados por objetivos terminales. La variable dependiente (VD) viene dado por las disposiciones positivas hacia las relaciones de confianza. La variable de control (VC) son pobladores de zona rural y urbana asociados, Caraz, Huaylas, Ancash, Perú.

Se evaluó antes y después de aplicar la VI con los instrumentos: Cuestionario de Conocimiento sobre los saberes de constructos económicos de gestión y la Escala de percepción hacia la disposición de relaciones de confianza para la generación de negocios a 24 integrantes que participaron de manera permanente a ocho talleres. La información se procesó con los estadísticos no paramétricos a fin de realizar los contrastes de hipótesis y estadísticos descriptivos para las actitudes hacia la confianza y la prueba de conocimientos.

\section{MARCO TEÓRICO Y DEFINICIÓN DE CONCEPTOS.}

Li, Bai y Xi (2012) refieren antecedentes contextuales de la confianza en la organización y comportamientos organizacionales centrándose en los efectos de los tres antecedentes contextuales más críticos: papel de liderazgo, el estado estructural y la norma cultural a nivel organizacional, considerando la confianza en la organización como un mediador entre los diferentes niveles. Los resultados empíricos se basan en un modelo jerárquico lineal con apoyo multidimensional del modelo a nivel cruzado de enlace de contexto-confianza-comportamiento. La muestra fue de 444 empleados de 82 empresas en China. La investigación sobre la confianza en la organización es tratada como un fenómeno entre los diferentes niveles y mediante la especificación de sus antecedentes y consecuencias contextuales básicas de comportamiento.

Yang (2005) señala que la literatura reciente muestra que los efectos de la confianza sobre los resultados en el lugar de trabajo tienen incidencias importantes, concluyendo que los aumentos en la confianza tanto cognitivo y afectivo en el supervisor reducen las relaciones de conflicto entre supervisor y subordinado. La confianza en la dirección afectiva puede ser un indicador importante de dedicación afectiva en la organización, siendo en esta dirección un indicador para el pronóstico en el rendimiento de la tarea. En las interacciones entre subordinados, tan demostrado por la comunicación abierta, se han encontrado estar influidos por la confianza en el supervisor. De manera particular, tanto la dirección cognitiva y afectiva de la confianza en el supervisor aúnan para el pronóstico de tarea y comportamiento de ciudadanía interpersonal centrada hacia compañeros de trabajo. Comparado con la confianza cognitiva, la confianza afectiva en el supervisor era más predictiva en la comunicación organizacional tanto en los canales hacia arriba como lateral. 
Sitter (2004) encuentra que en una organización la habilidad de un jefe de valorar y expresar la emoción es decisiva en el desarrollo de la confianza emoción de un empleado a su jefe.

Paredes (2010) concluye que la habilidad interpersonal para la negociación difiere significativamente según los estilos de vida adquiridos y por el nivel ocupacional de la población en empresas productoras en el Perú, excepto en el factor de control emocional de las habilidades interpersonales para la negociación. Teniendo en consideración que este factor emocional se comporta similarmente en todos los niveles ocupacionales a pesar de la posición desempeñada que demanda socialización en la organización, así como el tener en cuenta los grados saludables en el logro en la vida que tenga una persona, juega rol importante el coeficiente emocional (Baron en Goleman, 1996), ello orienta a plantear que las emociones, al tener un componente fisiológico, escapan al control racional y social, es decir, la emoción está vinculada a otros factores o procesos psicológicos, especialmente a la naturaleza psiconeurofisiológica de la personalidad.

El Estilo de vida también se va construyendo en un medio laboral, caracterizado por la complejidad como por la naturaleza de toda actividad de trabajo, el que se va desvinculando de la fuerza física, tal como es el caso de la gestión, el que genera espacios contextuales específicos donde sus actores entretejen una gama de afectos que cualifican el proceso de socialización.

Así, la confianza al ser un estado como producto del aprendizaje en el medio socio-cultural, este se va desarrollando en aspectos afectivos, prioritariamente con códigos identificados socialmente para una convivencia laboral. Entendiéndose por confianza al constructo que describe un estado psicológico para comprender las expectativas seguras sobre el comportamiento y las intenciones de otro, y como el componente fundamental en la generación de vínculos productivos que a la vez promueven un clima organizacional enriquecedor o saludable. El tipo de Estilo Vida Activo agresivo objetivo identificado en la población de colaboradores en el sector empresarial productivo (Paredes, 2010), y el más significativo en nuestra realidad (Vicuña y cols., 2000), que se caracteriza porque enfrenta la realidad, empleando los factores de control emocional, empatía, comunicación, perspectiva e involucramiento de la habilidad para la negociación, que contribuye eficazmente a una convivencia laboral sana.

Planteándose la confianza como un activo pragmático, tangible y que puede ser creado con mayor facilidad de la que se cree (Covey, 2011), esta orienta a encaminar programas dirigidos al aprendizaje para la gestión en vías de implementarlo en la cultura organizacional, a fin de que se constituya en un medio para el Estilo de Vida del colaborador cual fuera su posición laboral.

Rus \& Iglič (2005) al estudiar el nivel de confianza que los empresarios tienen en las instituciones y en los socios de negocios, encuentran que ella influye en la elección de mecanismos de gobernabilidad que coordinan las actividades económicas entre los actores, de pequeñas y medianas empresas en Eslovenia y Bosnia-Herzegovina, 
donde el entorno institucional es una variable que genera confianza en los negocios. El caso de Eslovenia ha permitido a sus actores basar sus relaciones de negocios en la confianza más que en el contrato, pero se encuentra que cuando el entorno institucional es débil se genera menos confianza, lo que conduce a los actores a basar sus relaciones económicas en contratos, y cuando los actores se apoyan en la confianza, a menudo, se trata de confianza institucional y no interpersonal.

Urbano, Toledano \& Ribeiro-Soriano (2011) mediante estudios de casos, revelan el papel fundamental que desempeñan las relaciones de confianza generadas entre los miembros de tres pymes españolas. Señalan que una de las claves para su éxito es el contexto de confianza para la gestión del personal en las empresas de reducida dimensión, lo que contribuye a arriesgarse a proponer y desarrollar nuevas iniciativas, aun más en un contexto de crisis económica en el periodo depresivo que se está viviendo en España desde 2008.

\section{Concepto de confianza}

Constructo que describe un estado psicológico para comprender las expectativas seguras sobre el comportamiento y las intenciones del otro, y componente fundamental en la generación de vínculos productivos que a la vez promueven un clima organizacional enriquecedor o saludable. El mismo es medido a través de los siguientes cuatro factores:

\section{Competencia}

Es una dimensión imprescindible para la obtención de resultados, de modo tal que el fracaso reiterado conduce a la pérdida de seguidores y de aliados o socios. Genera admiración y da esperanzas a los compañeros o seguidores, originando líderes efectivos en función de la situación para un caso pero no para otros, y que incluso son mejores en un nivel que en otro. Es aplicable en este caso la ley de Peter que dice que todo empleado en una jerarquía asciende hasta alcanzar su nivel de incompetencia, pero toda ley tiene sus excepciones. La competencia es un componente que asegura que se alcanzará la meta u objetivo por encima del producto del azar y merced a las destrezas de los gestores.

\section{Preocupación por el otro}

Entendido como benevolencia, es la elección del socio a partir de conductas de preocupación por los demás, no solo para asegurar la solución de un problema si es que se presenta, sino, también, para prevenirlo. Es lo contrario de pensar solo en uno mismo, dejar estancado al socio, o de no cumplir con la palabra. Se expresa en un interés consciente por tratar al otro como una persona y no como un simple generador de resultados.

\section{Equidad}

Es la distribución de medios en correspondencia al esfuerzo realizado en la ejecución de una tarea. Genera el sentimiento de ser tratado de manera justa y conduce a un estado de seguridad y de esperanza en la retribución. La equidad se basa en códigos capaces de orientar la subjetividad hacia la intersubjetividad sustentada como modus de vida del contexto social, es decir, legitimizando de manera evidente el valor organizacional. 
Mildred Paredes T., Héctor Hernández V., Renato Santivañez O., Miguel Iturrizaga A.

\section{Visión de sí mismo}

Describe la disposición autopercibida a sentirse merecedor de confianza por parte de otros, con capacidad de ser elegido como socio por las mismas condiciones que atribuye a sus potenciales socios. Para lograr tener un socio, debe considerarse a Mínguez (2011), "la confianza en los demás, tanto en las relaciones personales como entre organizaciones, implica la existencia de una adecuada gestión de las expectativas. Es en este ámbito donde se visualiza la confianza de forma que, para parecer confiable, se deben gestionar tanto las expectativas que los demás depositan en nosotros como las que nosotros depositamos en los demás".

\section{Constructo de socialización económica en gestión de negocios}

Se denomina así al proceso de adquirir fortalezas y competencias para la convivencia con bienestar y con crecimiento económico que pone fin a la pobreza extrema, reduce la exclusión social y es un medio ambientalmente saludable en el manejo eficiente y sostenible de recursos económicos, tangibles e intangibles. Entre sus fundamentos, son los procesos de gestión un medio para el logro de objetivos y la satisfacción de los involucrados, los que son a la vez gestores y clientes o usuarios, según su posición en un sistema organizacional.

Se expresa en los siguientes constructos:

\section{Sistema}

Es toda organización social, de ahí, las organizaciones son grupos sociales integrados por personas que realizan tareas y se valen de la administración, en cuyo proceso forman una estructura sistemática de relaciones de interacción, tendientes a producir bienes y/o servicios para satisfacer las necesidades demandadas por una comunidad dentro de un entorno que puede ser próximo como lejano y así poder satisfacer su propósito distintivo que está previsto en su misión. Es decir, la organización viene a ser un conjunto de actividades que prioriza la interacción de manera consciente y coordinada, formado por dos o más personas donde la cooperación entre ellas es esencial para su existencia. Para poder encaminar a la organización son necesarios:

\section{Gestión}

Es la actividad empresarial fundamental y transcendental de toda organización que busca, a través de personas como directores institucionales, gerentes, productores, consultores y expertos, dirigir y mejorar tanto la calidad vida como la productividad y por ende la competitividad de las organizaciones, sean empresas o negocios o instituciones. Una óptima gestión no busca solo hacer las cosas mejor, lo más importante es hacer mejor las cosas correctas y en ese sentido es necesario identificar los factores que influyen en el éxito o mejor resultado de la gestión.

\section{Estrategia}

La estrategia es un medio para solucionar objetivos y satisfacer la necesidad de una población o mercado mediante la búsqueda deliberada de un plan de acción que desarrolle la ventaja competitiva de una organización o negocio para obtener los objetivos trazados con recursos previstos. 
PRogRAMA de SOCIALIZACIÓN ECONÓMICA EN GESTIÓN NEGOCIOS Y RELACIONES DE CONFIANZA...

\section{Proceso Administrativo}

Para cumplir su misión la organización se vale de la administración, que tiene como proceso planificar, organizar, dirigir, evaluar y coordinar las actividades de los miembros de la organización y el empleo de todos los demás recursos organizacionales con el propósito de alcanzar las metas establecidas para la organización.

\section{Proyecto}

Es un producto profesional, técnico, orgánico y sistemático que expresa la solución de un problema. En sí, es un intento para llegar a un fin específico. Esto es posible gracias a tareas interrelacionadas y al uso correcto de los recursos con los que se cuenta. Los proyectos están delimitados por cuatro fases en relación al cliente o usuario de la organización, las cuales son:

1) Alcance: Es el trabajo a realizar con la finalidad de que el cliente quede satisfecho con el cumplimiento de los criterios de aceptación, las entregas y con los requisitos acordados al inicio del proyecto.

2) Costo: Es la cantidad convenida por el cliente a pagar por resultados aceptables del proyecto.

3) Programa: Relación de tiempos que dispone los momentos específicos para el comienzo y la culminación de cada tarea o actividad.

4) Objetivo: El objetivo de cualquier proyecto es el complementar el alcance dentro del presupuesto establecido y en la fecha determinada por el cliente. Para esto es importante que se desarrolle un plan antes de comenzar el proyecto, el cual debe de incluir todas las actividades y tareas, así como los costos y estimados de tiempo que se piensa se necesitará para la culminación del proyecto.

\section{Planeación}

Es una actividad del proceso administrativo que consiste en la selección de objetivos, alternativas, recursos y medios para alcanzar mayores niveles de desarrollo de un país, institución o empresa que se trate. Comprende en la práctica desde el nivel de formulación de ideas, pasando por el diseño de objetivos, metas, estrategias, políticas y programas, hasta los procedimientos, teniendo en consideración al sistema organizacional en perspectiva futura.

\section{Organización}

Es una actividad del proceso administrativo centrado en lograr la relación y la jerarquía de posiciones que se establecen entre los recursos humanos y velar por los diversos recursos, tal como los económicos de los que dispone la empresa para alcanzar los objetivos y metas propuestas en el plan correspondiente. Implica su competencia la implementación de una estructura de funciones, determinación de actividades requeridas para alcanzar las metas y objetivos de una empresa y de cada una de sus partes.

\section{Dirección}

Es una actividad del proceso administrativo, consiste en hacer que los miembros de la organización contribuyan a alcanzar los objetivos como responsabilidad de su posición, 
función inherente al gerente o ejecutivo que se logre, como acción de permamente motivación. La gestión significa creación, aporte, imaginación, iniciativa y criterio direccional para tomar decisiones acertadas que llevan a la organización a la consecución de sus objetivos con la percepción de líder en la relación de liderazgo en la estructura organizacional.

\section{Evaluación}

Es una actividad del proceso administrativo que consiste en aproximarse a la realidad del desempeño en el trabajo vinculado a la eficiencia y satisfación del trabajador o colaborador. Se emplea para medir el desempeño laboral en relación con las metas, detecta las desviaciones negativas y plantea las correcciones correspondientes en perspectiva de cumplir con los planes, mediante el feedback; llevando a un proceso de retroalimentación permanente que genera mejoras en los procesos de trabajo y salud ocupacional.

\section{Coordinación}

Es una actividad del proceso administrativo, como función esencial de la administración. Las acciones en este proceso permiten armonizar esfuerzos individuales y grupales encaminándolos hacia la consecución de objetivos, de forma eficiente, evitando la duplicidad de funciones y propiciando la calidad en el ejercicio de la posición del colaborador, favoreciendo la interdependencia del trabajo en equipo y el compromiso organizacional. También aúna de manera eficaz a gestionar de manera más adecuada la información de cada unidad de cada empresa y evitar rumores o alguna estructura informal en la gestión.

De lo anterior, se hace pertinente el planteamiento de hipótesis a ser confrontado mediante la investigación teniendo el propósito de instrumentar planes y programas asertivos para lograr mejorar su situación económica y, por ende, la inclusión con una mejora en la condición de vida de los colaboradores.

\section{Hipótesis demostrada en el estudio}

$\mathrm{H}_{\mathrm{i}}$ : El Programa de Socialización económica en gestión de negocios influye en las Relaciones de confianza en pobladores de zona urbana y rural asociados en Caraz, Huaylas, Ancash.

\section{Método y técnicas utilizadas}

Investigación del tipo experimental, con un diseño cuasiexperimental de evaluación antes-después para identificar el efecto de un Programa de Socialización económica que promueve la gestión de negocios en las Relaciones de confianza en pobladores de zona urbana y rural asociados en Caraz, Huaylas, Ancash.

\section{Población y muestra}

Los pobladores mayores de 16 años de los barrios de Caraz de ambos sexos La muestra fue determinada por la aceptación de los 32 colaboradores voluntarios, inicialmente que respondieron el cuestionario de Socialización económica en gestión de negocios, a quienes previamente se les informó del proyecto. 
PRogRAmA de SOCIALIZACIÓN ECONÓMICA EN GESTIÓN NEGOCIOS Y RELACIONES DE CONFIANZA...

La evaluación sobre la disposición hacia las relaciones de confianza fue respondida por 24 colaboradores, con una muerte experimental de 8 sujetos.

Con la asistencia de 24 colaboradores a los 8 talleres que corresponden al Programa de Socialización Económica en Gestión de Negocios (Variable Independiente) se realizó la evaluación de salida de la Disposición hacia las relaciones de confianza.

\section{Diseño de la investigación}

La investigación tiene diseño cuasiexperimental en donde se realizó una medición antes y después entre un periodo de tres meses mínimo, con los instrumentos para la medición previa y luego de aplicar los ocho talleres de capacitación. Asimismo, se utilizó en la instrumentalización, como parte del Programa de Socialización económica en gestión y las relaciones de confianza, talleres de capacitación en procesos administrativos instrumentado por casos y dinámicas de grupo para que cognitivamente puedan observar un modelo de un negocio o proyecto de negocio productivo de un bien o servicio, en torno a un producto propio del lugar. Los mismos son el contenido de los módulos del Programa de Capacitación en conceptos de economía para la gestión de calidad mediante la estrategia por interdependencias empresariales, con un soporte de Cartillas, tipo comic.

\section{Instrumentos de recolección de datos}

\section{Cuestionario de socialización económica en gestión de negocios}

El cuestionario tiene por objetivo conocer los saberes previos de los participantes del presente estudio referidos a conocimientos sobre gestión de negocios. La información prevista por este instrumento da cuenta de los aspectos de sistema, gestión y estrategia, objetivos, proceso administrativo, programa, planificación, organización, dirección, evaluación y coordinación.

Escala de disposiciones a las relaciones de confianza para la generación de negocios en pobladores asociados de la zona urbana y rural en Caraz, Huaylas, Ancash.

Escala tipo Likert, constituida por 20 ítems, distribuidos en cuatro factores: Competencia, Preocupación por el otro, Equidad y Visión de sí mismo, tal como se señala en la Tabla 1. Su Confiabilidad se realiza mediante el método de consistencia interna, el que se determinó mediante el coeficiente de Alfa de Cronbach con un valor de 0.738 .

Tabla 1. Tabla de Especificaciones Escala de Disposiciones a las Relaciones de Confianza en Negocios

\begin{tabular}{|c|c|c|}
\hline Factor & Dimensión & Indicador \\
\hline $\begin{array}{l}\text { Competencia: "grupo de } \\
\text { habilidades, competencias y } \\
\text { características que permitan a } \\
\text { la parte que tienen influencia } \\
\text { dentro de un dominio } \\
\text { específico" (Mayer et al., } \\
\text { 1995, p. } 717 \text { ). }\end{array}$ & $\begin{array}{l}\text { Capacidad, habilidad, } \\
\text { destreza, dominio y } \\
\text { experiencia percibida al } \\
\text { efectuar una actividad } \\
\text { determinada. }\end{array}$ & $\begin{array}{l}\text { Capaz de realizar la tarea. } \\
\text { Tener éxito en las cosas que } \\
\text { trata de hacer. } \\
\text { Conocimiento sobre el trabajo } \\
\text { que necesita hacerse. }\end{array}$ \\
\hline
\end{tabular}


Mildred Paredes T., Héctor Hernández V., Renato Santivañez O., Miguel Iturrizaga A.

\begin{tabular}{|c|c|c|}
\hline $\begin{array}{l}\text { La preocupación por el otro, } \\
\text { "la medida en que se cree que } \\
\text { la otra persona quiere hacer } \\
\text { el bien a uno, al margen de } \\
\text { un afán de lucro egocéntrico" } \\
\text { (Mayer et al., 1995, p. 718.). }\end{array}$ & $\begin{array}{l}\text { La lealtad, la franqueza, } \\
\text { el cuidado, la receptividad } \\
\text { disponibilidad para el } \\
\text { servicio, con evidencia de ser } \\
\text { benevolente. }\end{array}$ & $\begin{array}{l}\text { Está muy preocupado por mi } \\
\text { bienestar. } \\
\text { Mis necesidades y deseos son } \\
\text { muy importantes para el socio. } \\
\text { El socio se esforzará por } \\
\text { ayudarme. }\end{array}$ \\
\hline $\begin{array}{l}\text { Equidad: sentir en la } \\
\text { interacción Integridad, como } \\
\text { "la percepción de que el } \\
\text { fiduciario se adhiere a un } \\
\text { conjunto de principios que } \\
\text { el fideicomitente encuentra } \\
\text { aceptable" (Mayer et al., 1995, } \\
\text { p. } 719 \text { ). }\end{array}$ & $\begin{array}{l}\text { Percepción de } \\
\text { correspondencia y justicia, la } \\
\text { coherencia, el cumplimiento } \\
\text { la promesa, la fiabilidad, } \\
\text { la congruencia de valores, } \\
\text { discreción en las relaciones } \\
\text { interpersonales y económicas. }\end{array}$ & $\begin{array}{l}\text { El socio tiene un fuerte sentido } \\
\text { de la justicia. } \\
\text { El socio y los comportamientos } \\
\text { son muy consistentes. }\end{array}$ \\
\hline
\end{tabular}

Visión de sí mismo: "el desarrollo de apegos seguros hacia las otras personas manifiesto en la convicción de su capacidad" Bowlby, Erikson, Adler
La convicción de merecer ser elegido como socio por las propias cualidades y de tener buena capacidad para elegir socios y recursos para manejar en situaciones potencialmente frustrantes.
Sentirse elegible como socio. Considerar como necesario tener socios.

\section{Técnicas de dinámicas de grupo para el desarrollo de cada Módulo}

Según el contenido de cada taller del programa de intervención, se ha empleado como instrumento las técnicas de dinámica de grupo en los talleres, como medio vivencial en los ocho módulos guiado por objetivos terminales, referido al tema de socialización económica en gestión de unidades productivas de dimensión menor (pymes), reforzado con cartillas tipo Comic.

Los conceptos en economía para la gestión se han impartido y observado durante el desarrollo de los talleres del Programa de Socialización Económica aplicado o transferido según el modelo de la propia unidad productiva del colaborador, de ser posible centrado en el rescate de los productos culturales para su comercialización, basado en las expresiones culturales en paquetes comerciables.

\section{RESULTADOS}

\section{Análisis Estadístico del Cuestionario de Socialización Económica en Gestión de Negocios}

\section{Análisis descriptivo}

En la Tabla 2 se muestra los resultados descriptivos referidos al cuestionario de socialización económica en gestión de negocios en pobladores urbanos y rurales 
PRogRAMA de SOCIALIZACIÓN ECONÓMICA EN GESTIÓN NEGOCIOS Y RELACIONES DE CONFIANZA...

de Caraz-Huaylas, Ancash, en el que las puntuaciones directas son convertidas a porcentajes para facilitar su interpretación. Se puede apreciar que los resultados promedio alcanzados de los evaluados en el mejor de los casos están en torno al $50 \%$ de conocimiento sobre gestión de negocios, siendo los aspectos más débiles el de Evaluación (25\%), Objetivos (34\%), y Gestión y estrategia (39\%). Las más altas puntuaciones corresponden a Dirección (59\%) y Organización (57)\%.

Tabla 2. Datos descriptivos del cuestionario de socialización económica en gestión de negocios.

\begin{tabular}{ccc}
\hline & $\mathrm{N}$ & $\mathrm{M}(\%)$ \\
\hline Sistema & 32 & 51 \\
Gestión y Estrategia & 32 & 39 \\
Objetivos & 32 & 34 \\
Proceso Administrativo & 32 & 53 \\
Programa & 32 & 50 \\
Planificación & 32 & 45 \\
Organización & 32 & 57 \\
Dirección & 32 & 59 \\
Evaluación & 32 & 25 \\
Coordinación & 32 & 50 \\
Total & 32 & 47 \\
\hline
\end{tabular}

\section{Descripción y Análisis Estadístico de las Disposiciones hacia las Relaciones de Confianza en Negocios}

La Tabla 3 presenta los estadísticos descriptivos de las pruebas de entrada y salida de la disposición hacia las relaciones de Confianza en los negocios. Observándose el total con tendencia alta (53) dentro de los valores 46-60.

Tabla 3. Datos descriptivos de media y desviación estándar de los factores de Disposiciones hacia las relaciones de confianza.

\begin{tabular}{cccccc}
\hline $\begin{array}{c}\text { Factores de la dimensión de } \\
\text { confianza }\end{array}$ & N & M & DS & $\begin{array}{c}\text { Valor } \\
\text { mínimo }\end{array}$ & $\begin{array}{c}\text { Valor } \\
\text { máximo }\end{array}$ \\
\hline Competencia Pre-test & 24 & 17,75 & 1,82 & 16 & 20 \\
Competencia Post-test & 24 & 19,00 & 1,62 & 17 & 21 \\
\hline Preocupación por el otro Pre-test & 24 & 9,50 & 1,53 & 8 & 12 \\
\hline Preocupación por el otro Post-test & 24 & 10,00 & 0,72 & 9 & 11 \\
Equidad Pre-test & 24 & 9,00 & 1,25 & 7 & 10
\end{tabular}


Mildred Paredes T., Héctor Hernández V., Renato Santivañez O., Miguel Iturrizaga A.

\begin{tabular}{|c|c|c|c|c|c|}
\hline Equidad Post-test & 24 & 8,00 & 1,25 & 7 & 10 \\
\hline Visión de sí mismo Pre-test & 24 & 16,50 & 2,93 & 14 & 21 \\
\hline Visión de sí mismo Post-test & 24 & 16,00 & 3,46 & 11 & 20 \\
\hline Total disposiciones Pre-test & 24 & 52,75 & 5,93 & 46 & 62 \\
\hline Total disposiciones Post-test & 24 & 53,00 & 5,82 & 46 & 60 \\
\hline
\end{tabular}

\section{Análisis inferencial}

El análisis de la normalidad mediante la prueba de Shapiro-Wilk para cada una de las dimensiones de la escala de disposiciones hacia las relaciones de confianza indica distribuciones no normales $(\mathrm{p}<0.05)$. Dado que los datos corresponden a la evaluación antes y después para una misma muestra es que se usará el estadístico no paramétrico para la diferencia de medias relacionadas, siendo el mismo la prueba de Wilcoxon. Los resultados se muestran en la Tabla 4.

Tabla 4. Prueba de los rangos con signo de Wilcoxon aplicada al contraste pre-test-pos-test de la escala de disposición a las relaciones de confianza en pobladores urbanos y rurales de CarazHuaylas, Ancash

\begin{tabular}{cccccc}
\hline & Competencia & $\begin{array}{c}\text { Preocupación } \\
\text { por el otro }\end{array}$ & Equidad & $\begin{array}{c}\text { Visión de sí } \\
\text { mismo }\end{array}$ & $\begin{array}{c}\text { Total } \\
\text { disposiciones }\end{array}$ \\
\hline Z & $-2,107$ & $-1,257$ & $-3,464$ & $-1,086$ &, 000 \\
$\begin{array}{c}\text { Sig. asintót. } \\
\text { (bilateral) }\end{array}$ &, 035 &, 209 &, 001 &, 278 & 1,000 \\
\hline
\end{tabular}

\section{INTERPRETACIÓN DE RESULTADOS}

1. De acuerdo con los resultados expuestos en la tabla 4 se encuentra:

a. Diferencia significativa en la dimensión de Competencia, en términos de incremento.

b. Diferencia significativa en la dimensión Equidad, en términos de disminución.

c. No hay diferencia en cuanto a la preocupación por el otro (Benevolencia) y visión de sí mismo.

d. No se encuentra diferencia en el resultado total de la escala.

2. Con respecto a la confianza, un constructo definido a partir de cuatro componentes, competencia, preocupación por el otro, equidad y visión de sí mismo se pueden señalar que se satisfacen los siguientes supuestos:

a. La confianza puede darse aun cuando los cuatro componentes presentan niveles diferentes. En este caso afirmaremos que el conocimiento incrementa la importancia del factor de competencia y que la equidad es afectada por aspectos 
PRogRAMA de SOCIALIZACIÓN ECONÓMICA EN GESTIÓN NEGOCIOS Y RELACIONES DE CONFIANZA...

externos, tal como sostienen Rus \& Ig1ič (2005). Estos autores postulan que el entorno interinstitucional por sus mecanismos de gobernabilidad que coordina las actividades económicas influye en la confianza que tienen los empresarios en las instituciones y en las elecciones de sus socios.

b. La visión de sí mismo es una referencia a la personalidad, la cual se conceptualiza según el enfoque de Alfred Adler bajo la noción de estilo de vida y complejo medios-fin; y más que un reflejo del entorno, es una proyección del nivel que cada uno de los otros tres factores componentes de la confianza tiene para el individuo; por ejemplo, la socialización económica favorecerá inicialmente el factor competencia o cognitivo antes que incidir en la equidad.

c. Bajo esta perspectiva la noción de equidad sería la más afectada por los aspectos coyunturales toda vez que está influenciada por los factores de gobernabilidad.

d. La diferencia significativa en una de las dimensiones del constructo confianza es la competencia, es decir que la población estudiada da prioridad a los factores cognitivos del socio antes que a la preocupación por el otro y la equidad. Resultaría en una justificación de la conducta capaz en sus aspectos técnicos y profesionales pero con falencias en el aspecto ético y humano, y que usualmente es expresado en "roba pero hace".

e. Se da la posibilidad que se establezcan alianzas al desarrollar la confianza por la percepción y disposición a la competencia, expresada en capacidad, "dominio" del socio, mediante la capacitación o desarrollo del recurso humano como responsabilidad de la unidad respectiva de la organización, cuyos programas se centren en identificar y evaluar factores para otorgar competencia en la posición que desempeña, así como coadyuvar a instrumentar a un emprendedor o gestor la toma de decisión en la elección del socio.

Fortaleza acreditada por la competencia, la que debería estar aunado como señala Li, Bai, y Xi (2012) con la norma cultural a nivel organizacional. Como en este estudio la equidad es percibida como aquel factor que disminuye significativamente la disposición hacia la confianza, la misma puede adquirirse implementando por acción de la gestión y operatividad de los procesos administrativos a propósito de incorporarla como un indicador destacado en la cultura organizacional.

f. La gestión organizacional que se sustente en la confianza, procurará conductas que encamine su comportamiento entre los diferentes niveles de posiciones o grupos ocupacionales procurando que, como un mediador, la equidad norme el principio de la gestión, ejecutado por los procesos administrativos en su conjunto e interactivamente en el sistema organizacional, puesto que la preocupación por el otro en especial la Visión de Sí Mismo son factores ceñidos más a la personalidad. De ahí que, además de la competencia, otra dimensión que marca diferencia, es la equidad, ya que entre los factores del constructo de las disposiciones hacia las relaciones de confianza, en el presente estudio se da una relación inversa con la tendencia a disminuir, atribuido a factores institucionales externos, quienes perciben inequidad en el trato con ellos y a la inestabilidad de políticas y 
normas, coincidente a lo encontrado por Rus \& Ig1ič (2005), en el que el entorno institucional es una variable que genera confianza en los negocios, de lo contrario optan, si hacen negocio, por los contratos.

g. Si bien la confianza en los demás es influida fuertemente por el propio desarrollo personal y la estructura afectiva forjada desde la infancia, ya que interviene la confianza en sí misma, sería susceptible de presentar modificaciones favorables a partir de la constatación de las características específicas del entorno bajo la forma de microdecisiones, que todo colaborador en una organización va haciendo y va logrando.

3. En la aplicación práctica de los resultados de la investigación, se ha observado en los participantes de programas de socialización económica en negocios que visualizan lograr confianza para tener un socio en la dimensión de competencia, por ser adquirida mediante el aprendizaje y el intercambio de experiencias. Por lo tanto, se recomienda desarrollar programas de socialización económica en negocios estructurados según su rubro en los que el aspecto vivencial sea enfatizado como modalidad de capacitación.

\section{CONCLUSIONES}

1. Sobre la hipótesis de la investigación El Programa de Socialización económica en gestión de negocios influye en las Relaciones de confianza en pobladores de zona urbana y rural asociados en Caraz, Huaylas, Ancash. Se llega a las siguientes conclusiones.

a) Se incrementa significativamente la dimensión Competencia.

b) Se mantiene sin cambios la dimensión Preocupación por el otro.

c) La dimensión equidad presenta diferencia significativa pero en dirección negativa.

d) La dimensión visión de sí mismo se mantiene sin cambios.

2. En cuanto al Programa de Socialización económica se encuentra un nivel total que alcanza el $47 \%$ de la puntuación total. Siendo los puntos más débiles la evaluación (25\%), el Proceso Administrativo (34\%), y la Gestión y Estrategia (39\%) y los mejores puntos la Dirección (59\%) y la Organización (59)\%.

3. Por tanto se concluye que el Programa de Socialización económica en gestión de negocios influye parcialmente en las Relaciones de confianza en pobladores de zona urbana y rural asociados en Caraz, Huaylas, Ancash, por cuanto se han modificado dos de los factores que componen el constructo confianza.

\section{RECOMENDACIONES}

Replicar la presente investigación con pequeños y medianos empresarios asegurando una muestra más amplia y adecuando los instrumentos al contexto cultural.

Investigar la relación entre personalidad y componentes de la confianza como aporte al acervo teórico sobre el concepto de confianza y personalidad, en especial con la teoría. 
PRogRAMA de SOCIALIZACIÓN ECONÓMICA EN GESTIÓN NEGOCIOS Y RELACIONES DE CONFIANZA...

Incorporar el concepto de confianza como contenido curricular en los cursos vinculados al comportamiento organizacional y desarrollo personal social.

Ejecutar programas de socialización económica priorizando las características del socio competente pero además preocupado por los otros y equitativo.

Reconocimiento: De manera expresa al profesor Víctor Laguna por su disposición a la investigación y contacto con los colaboradores, a quienes agradecemos y reconocemos su cooperación en la construcción del conocimiento.

\section{REFERENCIAS BIBLIOGRÁFICAS}

Bowlby, J.(1999). Vínculo afectivos: Formación, desarrollo y pérdida. 3. ${ }^{a}$ ed. Madrid: Ediciones Morata, S.L.

Covey, S. (2011). La velocidad de la confianza. España: Paidós.

Kerlinger, F., y Lee, H. (2002). Investigación del Comportamiento. Métodos de Investigación en Ciencias Sociales. $4 .^{\mathrm{a}}$ ed. México: McGraw-Hill.

Goleman, D.(1996). Inteligencia Emocional. Barcelona: Editorial Cairos.

Guillen P. M. (2006). Ética en las organizaciones, Construyendo confianza. México. Pearson Educación, S.A., Prentice Hall.

Hernández,R.; Fernández, C.; y Baptista, P. (2007). Metodología de la investigación. 4. ${ }^{\mathrm{a}}$ ed. México: McGrawHill.

Johnson, D., \& Grayson, K. (2005). Cognitive and effective trust in service relationships. Journal of Business Research (58).

Li, Peter Ping; Bai, Yuntao; Xi, Youmin (2012). The Contextual Antecedents of Organizational Trust: A Multidimensional Cross-level Analysis. Management and Organization Review 8.2 (Jul 2012): 371. Document URL: http://search.proquest.com/ docview/1024130790?accountid=12268 Copyright: Copyright Blackwell Publishing Ltd. Jul 2012.

Martinez Fierro, S. (2003). Determining factors in partner's selection criteria in formation and development process in strategic alliances. Universidad de Cádiz (Spain). ProQuest Dissertations and Theses, 329 p. Retrieved from http://search.proquest.com/docview $/ 305221271$ ? accountid $=12268$.

Mínguez, V. Andrés (2011) La confianza como necesidad emocional”, en http:// marketing2puntoceu.wordpress.com/2011/05/21/la-confianza-como-necesidademocional/

Mayer, Roger C.; Davis James H. and Schoorman F. David (1995). An Integrative Model of Organizational Trust. The Academy of Management Review Vol. 20, N. ${ }^{\circ} 3$, Jul., 1995. page 711 of 709-734 https://www.jstor.org/action/exportSingleCitation?single Citation $=$ true $\&$ doi $=10.2307 \% 2 \mathrm{~F} 258792$ 
Papalia, D.E. Sterns, H. L.; Feldman, R. D. Camp, C. J. (2009). Desarrollo del adulto y vejez. 3. ${ }^{\mathrm{a}}$ ed. McGraw-Hill.

Paredes T., M. (2010). Las Habilidades Interpersonales para la Negociación según el Estilo de Vida y el Nivel Ocupacional en Empresas Productoras del Perú. Tesis de Doctorado en Psicología, Universidad Nacional Mayor de San Marcos, Escuela de Post Grado, Unidad de Post Grado de la Facultad de Psicología, Lima.

Paredes T., M. T.; Hernández V., Héctor M.;Vicuña P., A; Merino M. J. M. y col. (2010). Programa de Alfabetización Económica sobre conductas de emprendimiento en pobladores quechuas hablantes de Caseríos de Caraz. UNMSM/ CSI,IIPSI ITA-2010: Proyecto de Investigación Código: 101801011 Informe Técnico.

Paredes T., M. T.; Hernandez V., Héctor M.;Vicuña P., A.; Gonzalez Gil, C. M.y colb. (2012) Programa de socialización económica eninterdependencias empresarialespara el emprendimiento de turismo rural-vivencial en Caraz, Ancash. Revista de Investigación en Psicología, Lima Julio de 2012, Vol 15 N. ${ }^{\circ}$ 1, pp. 109-131, Instituto de Investigaciones Psicológicas de la Facultad de Psicología de la Universidad Nacional Mayor de San Marcos.

Paredes T., M. T.; Hernandez V., Héctor M.;Vicuña P., A.; Rivera B., J.C. y col. (2009) Socialización económica para el empoderamiento del caserío como unidad económica de la articulación organizacional de la Comunidad Campesina Cruz de Mayo, Caraz UNMSM/ CSI,IIPSI ITA-2011: Proyecto de Investigación 091801061: Informe Técnico.

Paredes T., M. T (2009). El Caserío es la unidad productiva en la articulación organizacional de zonas rurales altoandinas, Callejón de Huaylas, Ancash. Revista del Instituto de Investigaciones Económicas, Pensamiento Crítico N. ${ }^{o}$ 11:73-96 Junio, Lima,Perú.

Rus, A.; Ig1ič, H. (2005). Trust, Governance and Performance: The Role of Institutional and Interpersonal Trust in SME Development. International Sociology, 20(3), 371-391. doi:10.1177/0268580905055481.

Simpson, J. (2007). Psychological Foundations of Trust. Current Directions in Psychological Science, 16(5), 264-268.

Sitter, V. L. (2004). The effects of a leader's emotional intelligence on employees' trust in their leader and employee organizational citizenship behaviors. Regent University. ProQuest Dissertations and Theses, 165-165 pp. Retrieved from http://search.proquest. $\mathrm{com} /$ docview/305058665? accountid $=12268$. $(305058665)$

Urbano, D., Toledano, N. \& Ribeiro-Soriano, D. (2011). Prácticas de gestión de recursos humanos y desarrollo de nuevos proyectos innovadores: Un estudio de casos en las PYMEs*/Human resources management practices and corporate entrepreneurship: A case study in SMEs. Universia Business Review, (29), 116-130. Retrieved from http:// search.proquest.com/docview/868669498? accountid $=12268$ 
PRogRAMA de SOCIALIZACIÓN ECONÓMICA EN GESTIÓN NEGOCIOS Y RELACIONES DE CONFIANZA...

Vicuña, L.; Paredes M.; Hernández, H \& Solís, R. (2000). Relación entre los Complejos Medio-Fin con los tipos de afronte ante la crisis económica. Revista de Investigación en Psicología, Instituto de Investigaciones Psicológicas, Facultad de Psicología. UNMSM. Vol. 3(1), 95-114.

Yang, J. (2005).The role of trust in organizations: Do foci and bases matter? Louisiana State University and Agricultural \& Mechanical College). ProQuest Dissertations and Theses, 197-197 pp.

Retrievedfromhttp://search.proquest.com/docview/304992995 accountid=12268. (304992995). 